\title{
Design of PSS and SCRC Controllers to Damp out Low-Frequency Oscillations in Power System
}

\author{
Seung-Mook Baek \\ Division of Electrical, Electronic and Control Engineering, \\ Kongju National University, Cheonan, Chungnam, South Korea \\ Smbaek@kongju.ac.kr
}

\begin{abstract}
The paper describes a method to damp out low-frequency oscillations by design and tuning of both power system stabilizer (PSS) and controllable series capacitive reactance compensator (SCRC) controllers in power system. The PSS is generally used to mitigate the low-frequency oscillation by injecting supplementary signal to automatic voltage regulator (AVR) and the SCRC is used to enhance the transfer capacity for the transmission line in power system. Especially, the design of the external controller of the SCRC can provide effective damping for the low-frequency oscillation. In the paper, the effect of the design of both controllers on the system stability is analyzed. Also, the parameter tuning method is introduced and the performances of the proposed method are carried out by time-domain simulation with single-machine connected to infinite bus.
\end{abstract}

Keywords: Low-frequency oscillation, power system stabilizer, series capacitive reactance compensator, small signal stability, transient stability.

\section{Introduction}

In power system, the low-frequency damping problem has been attractive attention on the small signal stability researches. Especially, the modern power system becomes gradually large and the operating point of the system is close to the limitation of the system capacity. Therefore, the oscillation followed by disturbances can make the system unstable or collapsed. The power system stabilizer (PSS) is an important controller for damp out the low frequency oscillation. The PSS induces supplementary signal for the automatic voltage regulator (AVR) and excitation system in power system when the system is oscillated. Meanwhile, in Korea, installation and periodical adjustment of the PSS parameters in all the generators over 500 MVA capacity have been a strict rule in the system reliability regulation since 2005. Also, the dynamic behaviors of the PSS are affected by the linear parameters (gain and time constant of phase compensator). The appropriate selection of linear parameters is very important and has been usually made by using the conventional tuning techniques [1]-[2] based on the small signal stability analysis.

Among power-electronic-based controllers that control power flow in the power lines, the controllable series capacitive reactance compensator (SCRC), which is one of the series flexible AC transmission system (FACTS) devices, has attracted attention due to its rapid response control capability and the effective damping performance during disturbances [3]-[6]. For the rapid response control capability, the internal controller 
has been progressively developed to influence power flow as maintaining the injected voltage in quadrature with the line current and keeping the capacitor voltage constant. Otherwise, the external controller, which consists of the conventional linear controllers, is used to improve the low-frequency oscillation damping performance during disturbances [7].

On the other hand, the power system exhibits a mixed behavior of continuous dynamics and discrete-time dynamics. Interactions between continuous dynamics and discrete dynamics are an intrinsic part of power system dynamic behavior [8]. Devices that obey physical laws, which are generators and their controllers or parameters such as capacitors and inductors within power electronic circuits, typically lead continuous dynamics. On the other hand, even-driven discrete behaviors are associated with the protection devices, power electronics switches, and saturation limits in physical devices [9]. Therefore, the numerical modeling for the whole power system is not easy and the parameter selection of the components in power system also is a hard issue. In the paper, the power system with PSS and SCRC is modeled with differential and algebraic equations in order to catch the linear and nonlinear dynamics. Then, by making system matrix with analyzing those equations, the appropriate parameters of the PSS and SCRC are selected to improve low frequency oscillation damping.

In this paper, the design of the PSS and SCRC is proposed to improve small signal and transient stability in power system. That is, the external controller of the SCRC can be designed to bring more damping performance with action of the PSS though the SCRC can achieve its inherent control goal to improve transmission line capacity [7].

\section{Introduction of PSS and SCRC}

In this section, the main goals of the PSS and the SCRC are introduced with an explanation of their structure and use. The PSS is used for damp out the low frequency oscillation. And, the SCRC is used to enhance the transfer capacity for the transmission line in power system. Especially, the design of the external controller of the SCRC can provide effective damping for the low-frequency oscillation.

\subsection{Power System Stabilizer}

The PSS in Fig. 1 consists of five controllers, which are the gain, lowpass filter, washout filter, two phase-lead compensators, and output limits in order. Generally, the gain $\left(\mathrm{K}_{\mathrm{PSS}}\right)$ is set within the values between 2 to 10 . The lowpass filter is used to remove high frequency noise from the input signal and general value of its time constant $\mathrm{T}_{\mathrm{L}}$ is set to $0.01 \mathrm{~s}$. The washout filter is employed as a high-pass filter that removes dc component existing in the input signal. Its time constant, $\mathrm{T}_{\mathrm{W}}$ is generally set to $10 \mathrm{~s}$. Also, the phase-lead compensator provides damping to the system by compensating for the phase-lag between the PSS output $\left(\mathrm{V}_{\mathrm{PSS}}\right)$ and electrical torque. Its time constants, $\mathrm{T}_{1}$ to $\mathrm{T}_{4}$ are adjusted depending on system conditions [8], [10].

The PSS in Fig. 1 is connected to the AVR and inject supplementary signal at summing junction as shown in Fig. 1. Also, the AVR with PSS is connected to the generator and it controls the terminal voltage of the generator by adjusting the excitation voltage in rotor side of the generator. In general, in order to evaluate the performance of the PSS, a simple power system in which the generator is connected to the infinite bus is considered as illustrated in Fig. 2. The generator equipped with the AVR and the PSS is connected to the infinite bus by transmission system and some disturbances are applied to bring oscillation to power system. 


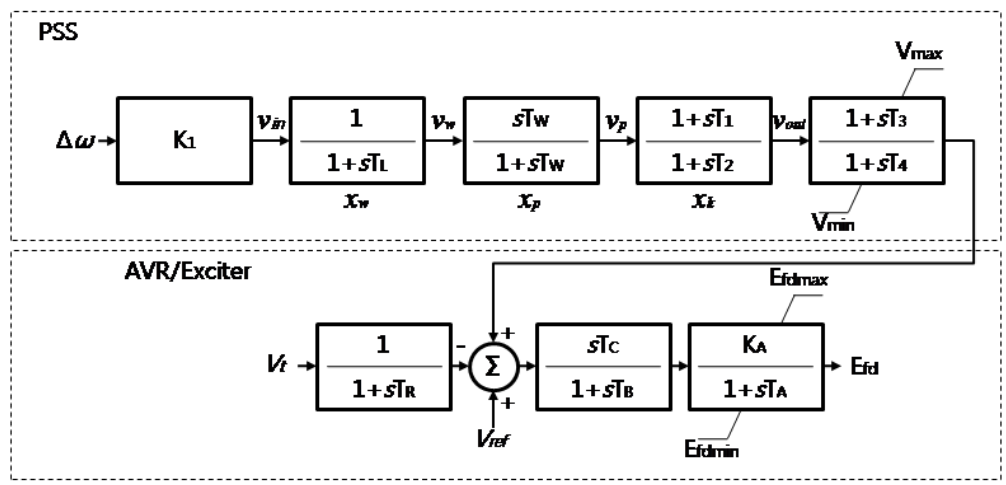

Figure 1 AVR/PSS Block Representation

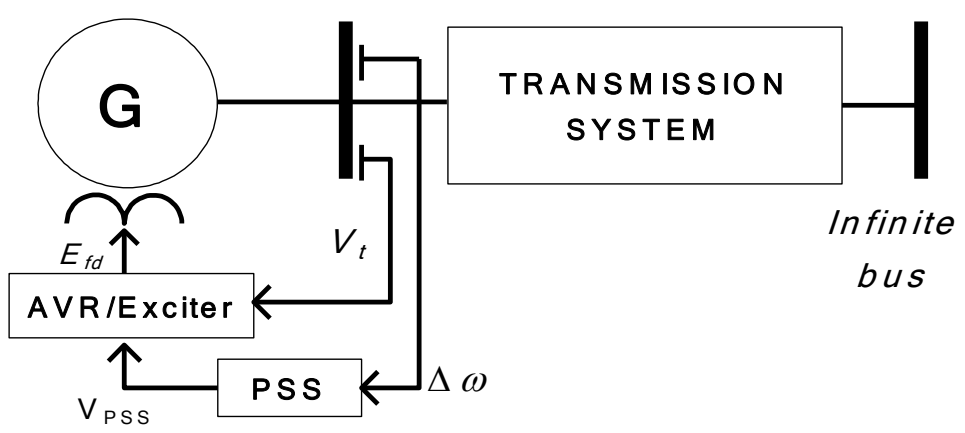

Figure 2 Single-Machine Connected to Infinite Bus (SMIB) System

\subsection{Series Capacitive Reactance Compensator}

The SCRC is one of the representative series FACTS devices, which are operated by gate turn-off thyristors (GTOs) based switching voltage-source pulse-width-modulated (PWM) inverter. There are two main controllers in operation of the SCRC. First is the internal controller which is shown as block diagram in Fig 3. The main objectives of the internal controller are to ensure that the injected voltage, $v_{c}$, at the AC terminal of the inverter is in quadrature with the transmission line current $i_{s}$ and to maintain the constant voltage $V_{d c}$ in steady state [7].

For the operation of line impedance compensation mode in the SCRC, the transmission line currents $i_{s a}, i_{s b}$, and $i_{s c}$ are first transformed by Park's transformation [1] into $i_{d}$ and $i_{q}$ which are $d$ and $q$ axes components in a synchronously rotating reference frame. Then, the peak value of the current vector is calculated and the desired magnitude of the compensating voltage vector is now determined by multiplying the magnitude of the current vector by the factor $2 \sqrt{2} / V_{d c}$ and the desired value of the capacitive reactance $X_{C}$. The result is the modulation index $m_{i}$ for the inverter [7 ].

Also, the internal controller of the SCRC in Fig. 3 has two feedback loops, which are an inner power control loop and an outer voltage control loop. The outer control loop ensures that no real power is exchanged between the inverter and the transmission line, that means that the line current leads the injected voltage by $90^{\circ}$ as well as that the DC capacitor voltage $V_{d c}$ is kept constant, as shown in Fig. 4. The inner feedback loop effectively reduces the gain required in the forward path of internal control to achieve the overall closed loop bandwidth desired in the SCRC. The advantage gained by the 
inclusion of the inner feedback loop is, therefore, that the necessary bandwidth of the phase-locked loop (PLL) can be achieved with less compromise in the response of the system to a disturbance on the DC side of the inverter. More details for the internal control of the SCRC are given in [12].

The objective of the external controller (EC) in Fig. 3 is to improve system damping performance by optimally modulating the reference of the internal controller. Design issues for the $\mathrm{EC}$ are discussed in this section. By adding an external control loop to the compensator, the system damping can be improved. However, a remaining open question is the selection of the input signals for the external loop. It is suggested in reference [6] that the speed deviation signal $\Delta \omega$ from a nearby generator could be used to generate the supplementary control signal $\Delta X_{C}$. In addition to $\Delta \omega$, the active power deviation signal $\Delta P_{S}$ is also considered as an input to the external controller as shown in Fig. 3. It can be seen from the generator swing equation [10],

$$
\Delta \dot{\omega}_{i}=\frac{1}{M_{i}}\left(\Delta P_{s i}-D_{i} \Delta \omega_{i}\right),(i=1, \cdots m)
$$

where $\Delta P_{S}$ is effectively the derivative of $\Delta \omega$. This is therefore a dual input-based controller design [7].

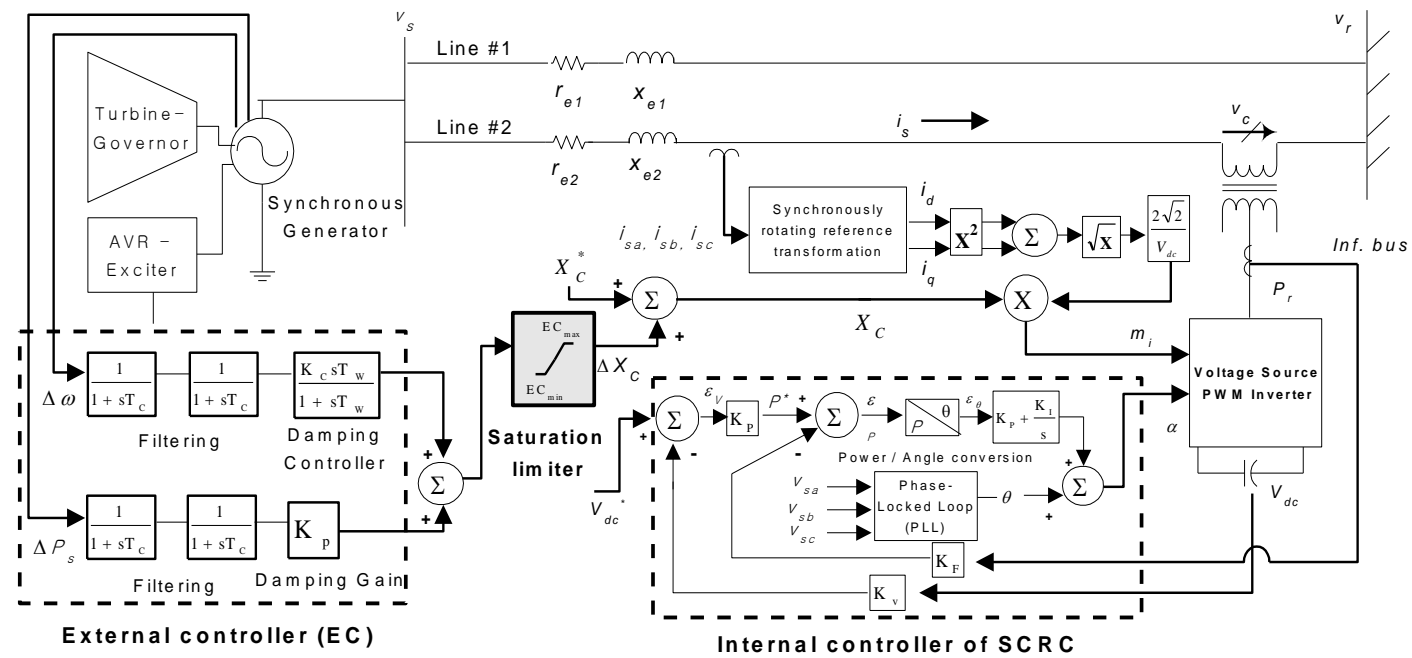

Figure 3 SCRC with Controllers

As mentioned before, the control outputs from the EC with high gains can cause an undesirable large-disturbance transient response in a physical system. To satisfy the constraints of physical systems and therefore to enforce practical limits resulting from the system restriction, the saturation limiter on the output of the EC is now considered, with non-smooth nonlinear dynamic behavior being the focus in this paper [7].

Moreover, the simulation study is carried out on the assumption that there is no communication delay incorporated in the simulation. In practice, the data communication of $\Delta P_{S}$ and $\Delta \omega$ to the SCRC from a remote location should be processed without significant time delays. In other words, these signals are required to 
communicate without any time delay or at least faster than a $0.2 \sim 0.5 \mathrm{~s}$ time delay for the successful damping of low-frequency oscillations. Ethernet can be considered as a real-time data communication system [7].

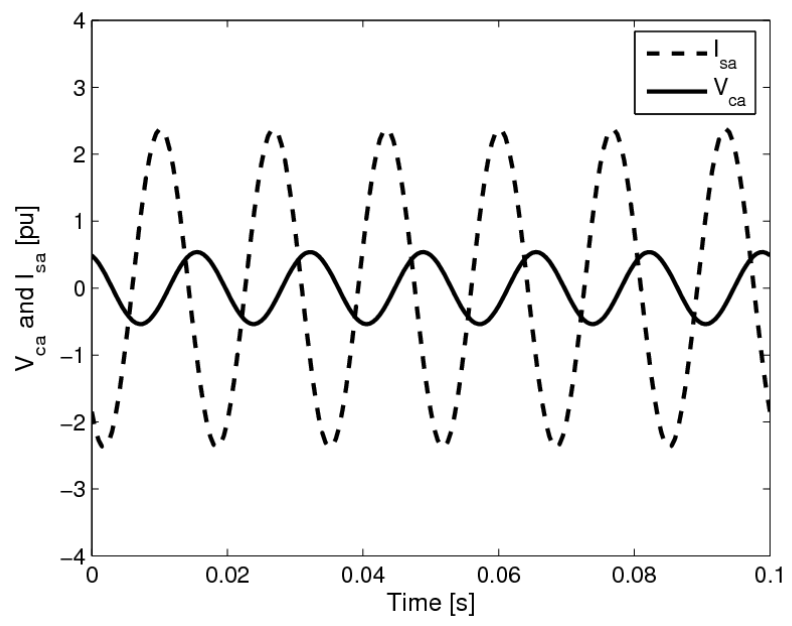

Figure 4 Injected Voltage $v_{c a}$ and Line Current $i_{s a}$ at Steady State $(t=5.9$ to 6 s)

\section{Power System Modeling}

As mentioned in the previous section, the behavior of the power system is characterized by the following:

- Continuous and discrete states.

- Continuous dynamics.

In other words, the power system needs to be modeled mathematically by consisting of the interactions between linear and nonlinear behaviors. Therefore, the power system modeling with the differential and algebraic equations can be presented without loss of generalities as follows [9].

$$
\begin{aligned}
& \underline{\dot{x}}=\underline{f}(\underline{x}, y) \\
& 0=g(\underline{x}, y)
\end{aligned}
$$

where $x$ represents the continuous dynamic states, for example generator angles, speed, and fluxes; $y$ represents algebraic states, e.g. load bus voltage magnitudes and angles.

With Eqs. (1) (2), the power system can be modeled as maintaining its nonlinear properties. The generator $(\mathbf{G})$ in Fig. 2 is accurately represented by a six-order machine model, viz., a two-axis $(d-q)$ model with two damper windings in each axis [9]-[10]. That means that six differential equations are needed to make mathematical model for the generator. More detailed explanations related to the SMIB system including the generator are given in [10]. Also, the differential and algebraic equations on the PSS and the EC of SCRC in Figs. 1 and 3, respectively, can be derived by Eq. (3) (6). The parameters in these equations are indicated in each model in Figs. 1 and 3. Especially, the value of $\Delta w$ in the Eq. (4) is from the generator model and the equations for generator and PSS are connected and applied simultaneously to simulate power system dynamics. 


$$
\begin{aligned}
f_{p s s}=\left\{\begin{array}{l}
\dot{x}_{w}=v_{w} / T_{w} \\
\dot{x}_{p}=T_{2} v_{p}-T_{1} v_{w} \\
\dot{x}_{k}=T_{4} v_{p}-T_{3} v_{k}
\end{array}\right. \\
g_{p s s}=\left\{\begin{array}{l}
\Delta w K_{1}-v_{w}-x_{w} \\
T_{2} v_{p}-T_{1} v_{w}-x_{p} \\
T_{4} v_{\text {out }}-T_{3} v_{p}-x_{k}
\end{array}\right. \\
f_{\text {SCRC }}=\left\{\begin{array}{l}
\dot{x}_{1}=y_{1} \cdot K_{C} / T_{C}-y_{2} / T_{C} \\
\dot{x}_{2}=y_{3} / T_{W} \\
\dot{x}_{3}=y_{4} \cdot K_{P} / T_{C}-y_{5} / T_{C} \\
\dot{x}_{4}=y_{3}+y_{5}-y_{6}
\end{array}\right. \\
g_{\text {SCRC }}=\left\{\begin{array}{l}
g_{1}=y_{2}-x_{1} \\
g_{2}=y_{2}-y_{3}-x_{2} \\
g_{3}=y_{3}-x_{5} \\
g_{4}=E C C_{\text {max }}-\Delta X_{\text {Cout }}-y_{8} \\
g_{5}=\Delta X_{\text {Cout }}-E C_{\text {min }} y_{9}
\end{array}\right.
\end{aligned}
$$

Once the whole system is analyzed and degraded by using Eqs. (1) (2), we can compute the system matrix in order to construct characteristic equations. The system matrix A can be effectively computed by the power system modeling with (1) (2), which provides the partial derivatives of $f$ and $g$ with respect to both the states, $x$ and algebraic variables, $y$. Thereafter, the eigenvalues of the overall system can be computed from the system matrix A with the reduced order in (7). Therefore, the specific eigenvalues correspond to the low-frequency oscillation mode in the power system can be extracted from the system matrix $\mathbf{A}$ [9].

$$
\begin{aligned}
\Delta \underline{\dot{x}} & =\underline{f}_{\underline{x}} \cdot \Delta \underline{x}+\underline{f}_{y} \cdot \Delta y, 0=g_{\underline{x}} \cdot \Delta \underline{x}+g_{y} \cdot \Delta y \\
\Delta y & =-g_{y}^{-1} \cdot g_{\underline{x}} \cdot \Delta \underline{x}, \Delta \underline{\dot{x}} \\
& =\left(\underline{f}_{\underline{x}}-\underline{f}_{y} \cdot g_{y}^{-1} \cdot g_{\underline{x}}\right) \cdot \Delta \underline{x}=\mathbf{A} \cdot \Delta \underline{x}
\end{aligned}
$$

\section{Appropriate Parameter Selection for PSS and SCRC}

By using Eq. (7), the eigenvalues correspond to the low-frequency oscillation are varied according to the changes of parameter values of the PSS and ES of SCRC. Since the real parts of the eigenvalues represent the damping ratio, we need to select the large value of those toward negative direction on complex plane.

Actually, the parameters which have high sensitive to the eigenvalue change are $K_{1}, T_{1}, T_{2}$, $\mathrm{T}_{3}$, and $\mathrm{T}_{4}$ for the PSS and $\mathrm{K}_{\mathrm{C}}$ and $\mathrm{K}_{\mathrm{P}}$ for the EC of the SCRC. Therefore, the appropriate parameter set for those value can be obtained by Eq. (7). Table I instructs the eigenvalues on both cases. For initial simulation, the tuned PSS is only applied without the SCRC. Otherwise, tuned PSS and SCRC are applied. As referring to Table I, the absolute values of the real part of the eigenvalues by the proposed method show twice larger than those for the initial ones. That means that the tuned parameters for PSS and SCRC bring more damping followed by disturbances than the initial ones and hence the small signal and transient stabilities in power system can be improved. 
Table I Parameters and Eigenvalues according to Application of PSS Only and Both

\begin{tabular}{|c|c|c|c|c|c|c|c|c|}
\hline \multirow{2}{*}{ Values } & \multicolumn{9}{|c|}{ PSS } & \multicolumn{2}{|c|}{ SCRC } & \multirow{2}{*}{ Eigenvalues } \\
\cline { 2 - 8 } & $\mathrm{K}_{1}$ & $\mathrm{~T}_{1}$ & $\mathrm{~T}_{2}$ & $\mathrm{~T}_{3}$ & $\mathrm{~T}_{4}$ & $\mathrm{~K}_{\mathrm{C}}$ & $\mathrm{K}_{\mathrm{P}}$ & \\
\hline PSS only & 2 & 0.25 & 0.025 & 0.25 & 0.025 & - & - & $-2.05 \pm j 8.70$ \\
\hline Both & 4.0 & 0.5 & 0.05 & 0.95 & 0.15 & 5.3 & 1.3 & $-4.21 \pm j 10.70$ \\
\hline
\end{tabular}

\section{Simulation Results}

In order to simulate the proposed control scheme, the paper uses Matlab software to build differential and algebraic equations for power system and its controllers including the PSS and SCRC. Thereafter, two tests are examined to show that the proposed method can improve small signal and transient stabilities.

To evaluate the performance on the small signal stability, the terminal voltage of the generator in Fig. 2 is heightened as 3\%. Figures 5 and 6 show terminal voltage response and real power output of the generator on the $3 \%$ step test, respectively. It is clearly shown that the proposed method can dramatically improve the small signal stability against the small disturbance. Also, the system can be stable fast if the well tuned PSS and SCRC are applied.

Also, power system in Fig. 2 is now disturbed by applying a $100 \mathrm{~ms}$ three-phase short circuit fault with the fault-impedance of $0.05 \mathrm{pu}$ to the generator terminal bus at 1 $\mathrm{s}$ to see the transient stability. The proposed method can bring good performances on the transient stability as shown in Figs. 7 and 8.

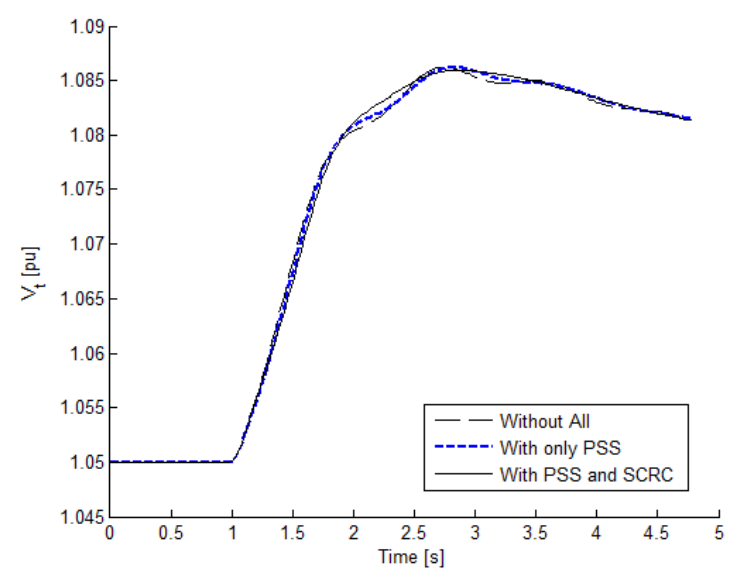

Figure 5 3\% Step Response: Terminal Voltage Response [pu] 


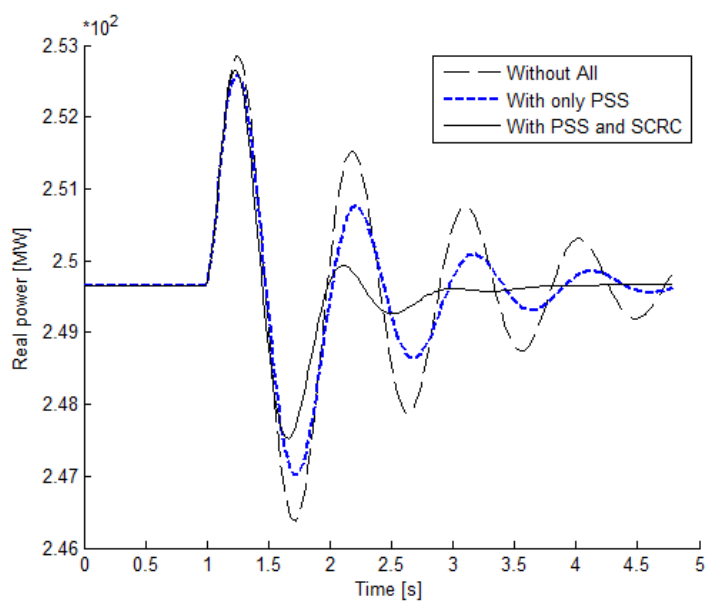

Figure 6 3\% Step Response: Real Power Response [MW]

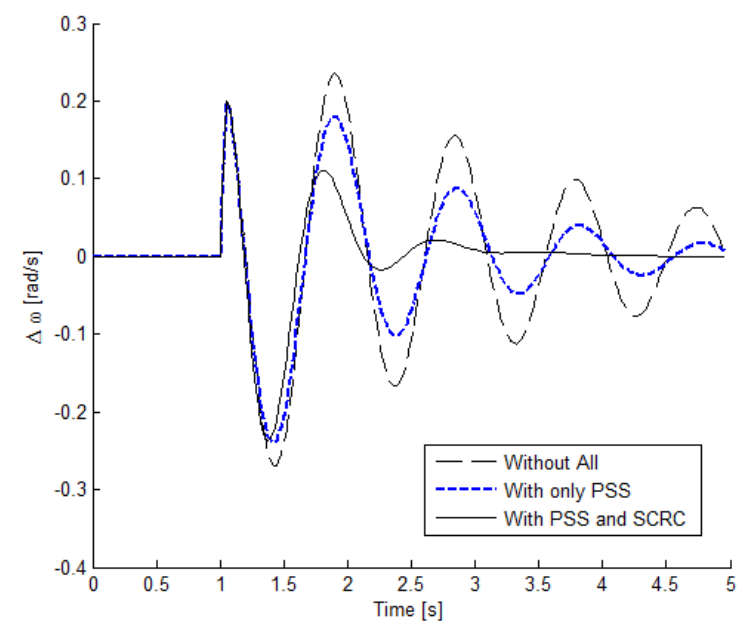

Figure 7 Three-phase Fault Response: Generator Speed Deviation [rad/s]

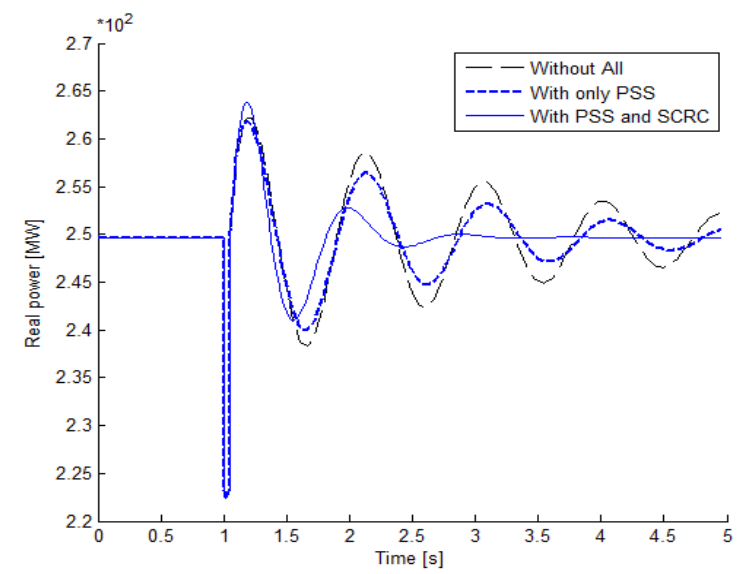

Figure 8 Three-Phase Fault Response: Real Power Response [MW] 


\section{Conclusions}

The paper described a method to damp out low-frequency oscillations by design and tuning of both power system stabilizer (PSS) and controllable series capacitive reactance compensator (SCRC) controllers in power system. The PSS is generally used to mitigate the low-frequency oscillation by inject supplementary signal to automatic voltage regulator (AVR) and the SCRC is used to enhance the transfer capacity for the transmission line in power system. In the paper, the effect of the design of both controllers on the system stability was analyzed. Also, the parameter tuning method was introduced.

The paper carried out two simulation tests to evaluate the performance of the proposed method. One is a small signal stability test and the other is transient stability test. By both tests, it was guaranteed that the proposed method could improve quite system stability.

\section{Acknowledgements}

This work was supported by a research grant from Kongju National University in 2013.

\section{References}

[1] J. H. Shin, J. G. Lee, S. C. Nam and Y. D. Choy et.al., "A Tuning Method for the Power System Stabilizer of a Large Thermal Power Plant and its Application to Real Power System : Part I - Selection of Parameters by Off-line Simulation”, Journal of the Korean Institute of Illuminating and Electrical Installation Engineers, vol. 23, no. 12, (2009) December, pp. 191-200.

[2] J. H. Shin, S. C. Nam, S. M. Baek and J. Y. Song et.al., "A Tuning Method for the Power System Stabilizer of a Large Thermal Power Plant and its Application to Real Power System: Part II - Field Tests and Verification of PSS Performance", Journal of the Korean Institute of Illuminating and Electrical Installation Engineers, vol. 25, no. 8, (2011) August, pp. 114-121.

[3] G. A. Adepoju and O. A. Lomolafe, "Analysis and Modelling of Static Synchronous Compensator (STATCOM): A comparison of Power Injection and Current Injection Models in Power Flow Study", IJAST, vol. 36, (2011) November.

[4] N. Farouk, "Proposal for Improve the Electrical Power Supply in Port Sudan Town”, IJAST, vol.42, (2012) May.

[5] H. Kouara, H. Laib and A. Chaghi, "A New Method to Extract Reference Currents for Shunt Active Power Filter in Three Phase Four Wire Systems", IJAST, vol.46, (2012) September.

[6] B. S. Rigby and R. G. Harley, "An improved control scheme for a series-capacitor reactance compensator based on a voltage-source inverter", IEEE Trans. Industry Application, vol. 34, (1998) March/April, pp. 355-363.

[7] S. M. Baek and J.-W. Park, "Optimal tuning for saturation limiter of a series FACTS", 6th IEEE International Conference on Industrial Informatics, (2008) July.

[8] S. M. Baek, J. W. Park and I. Hiskens, "Optimal Tuning for Linear and Nonlinear Parameters of Power System Stabilizers in Hybrid System Modeling”, IEEE Trans. on Industry Applications, vol. 45, no. 1, (2009) Jan./Feb., pp. 87-97.

[9] A. Van der Schaft and H. Schumacher, “An Introduction to Hybrid Dynamical Systems", SpringerVerlag, London, (2000).

[10] P. Kundur, "Power system stability and Control", EPRI Editors, McGraw-Hill, Inc. ISBN 0-07-035958$X,(1993)$.

[11] N. G. Hingorani and L. Gyugyi, "Understanding FACTS-concepts and technology of flexible ac transmission systems", IEEE Press, New York, (2000).

[12] B. S. Rigby, N. S. Chonco and R. G. Harley, "Analysis of a power oscillation damping scheme using a voltage-source inverter”, IEEE Trans. on Industry Applications, vol. 38, no. 4, (2002) July-August, pp. 1105-1113.

[13] J. W. Park and R. G. Harley, "Secondary control for a series reactive compensator based on a voltagesource PWM inverter”, IEEE PE Letters, vol. 2, no. 4, (2004) December, pp. 117-120. 


\section{Authors}

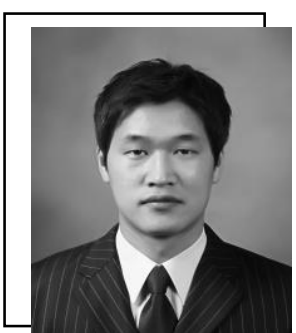

\section{Seung-Mook Baek}

He was born in Seoul, Korea. He received B.S., M.S., and Ph.D. degrees from the School of Electrical and Electronic Engineering, Yonsei University, Seoul, Korea, in 2006, 2007, and 2010 respectively, He is currently an Assistant Professor in the Division of Electrical, Electronic and Control Engineering, Kongju National University, Cheonan, Korea. He was a Research Engineer with KEPCO Research Institute, during 2009-2012. His current research interests are in power system dynamics, hybrid systems, optimization control algorithms, real-time simulation, flexible ac transmission system (FACTS) devices, and control of distributed generations. 